\title{
Criminologie
}

\section{Prévenues et détenues logées à la même enseigne, l'exemple des prisons de Burnaby et Tanguay}

\section{Julia McLean}

Volume 28, numéro 2, 1995

La détention provisoire

URI : https://id.erudit.org/iderudit/017372ar

DOI : https://doi.org/10.7202/017372ar

Aller au sommaire du numéro

\section{Éditeur(s)}

Les Presses de l'Université de Montréal

ISSN

0316-0041 (imprimé)

1492-1367 (numérique)

Découvrir la revue

Citer cet article

McLean, J. (1995). Prévenues et détenues logées à la même enseigne, l'exemple des prisons de Burnaby et Tanguay. Criminologie, 28(2), 43-60.

https://doi.org/10.7202/017372ar
Résumé de l'article

This article addresses the issue of the particular categories and degrees of constraints imposed upon pre-trial and convicted female inmates in Canadian institutions, focusing on two of those referred to as examples, Maison Tanguay in the province of Québec and the Burnaby Correctional Centre for Women located in British Columbia. It describes the conditions faced by women, incarcerated for a more or less long term period, and attempts to portray some reasoning as to why a pre-trial inmate must be subjected to similar or worse prison conditions as that of a convicted fellow. In order of do so, the author considers studies such as Biron (1992), Heidensohn (1985) and Bertrand (I994). The article concludes by indicating that due to the incertainty of her situation (would the suspect be convicted, when and what would be the sentence?) a pre-trial inmate may suffer additional constraints compared to an inmate convicted to prison for several years. 
This article addresses the issue of the particular categories and degrees of constraints imposed upon pre-trial and convicted female inmates in Canadian institutions, focusing on two of those referred to as examples, Maison Tanguay in the province of Québec and the Burnaby Correctional Centre for Women located in British Columbia. It describes the conditions faced by women, incarcerated for a more or less long term period, and attempts to portray some reasoning as to why a pre-trial inmate must be subjected to similar or worse prison conditions as that of a convicted fellow. In order of do so, the author considers studies such as Biron (1992), Heidensohn (1985) and Bertrand (1994). The article concludes by indicating that due to the incertainty of her situation (would the suspect be convicted, when and what would be the sentence?) a pre-trial inmate may suffer additional constraints compared to an inmate convicted to prison for several years.

\section{INTRODUCTION}

Des études de plus en plus nombreuses portent sur les conditions de vie des femmes en prison. Toutefois, généralement, elles ne font pas de différence entre prévenues et condamnées, puisque ces deux groupes de détenues se retrouvent quasi invariablement au sein d'une même institution. On fait alors, implicitement, l'équation selon laquelle les conditions de vie sont les mêmes pour toutes. Notre étude qui, à l'instar des autres, portait à l'origine sur les conditions de vie - plus précisément, dans notre cas, sur les contraintes - imposées aux détenues quel que soit leur statut (prévenues ou détenues), révèle des différences importantes qui soulignent la nécessité de distinguer entre ces deux groupes de détenues que sont, pour une part, les femmes soumises à une mesure de détention provisoire (celles incarcérées durant le temps de leur procès) et, pour une autre part, celles d'ores et déjà condamnées à des peines d'incarcération de plus ou moins

1. Les résultats traités dans le cadre de cet article sont issus d'une recherche subventionnée par le Conseil de recherche en sciences humaines du Canada.

2. L'auteure tient à remercier Mme Andrée Fagnan pour sa traduction éclairée du texte. 
longue durée. Car, s'il est vrai que ces femmes se trouvent regroupées à l'intérieur de la même enceinte, les conditions de vie et les contraintes qui leur sont imposées se révèlent dans bien des cas ou bien différentes (la différence jouant le plus souvent au détriment des prévenues) ou bien différemment vécues. C'est donc avec cette volonté de distinguer, pour une seule el rare fois, les conditions de vie et les contraintes imposées aux prévenues d'une part et aux détenues d'autre part, que nous abordons cet article. Il y sera question plus particulièrement de deux institutions carcérales canadiennes réservées aux femmes prévenues et condamnées: la Maison Tanguay et le Burnaby Correctional Center for Women. Nous jetterons, d'abord, un bref regard sur la situation de l'emprisonnement au Canada, histoire de situer d'une part la place des femmes incarcérées dans l'ensemble de la population carcérale canadienne et d'autre part la place respective qu'y occupent prévenues et condamnées.

\section{Méthodologie}

L'étude originale d'où sont tirées les données qui suivent s'est intéressée à vingt-quatre institutions pour femmes réparties dans huit pays différents (Canada, États-Unis, Allemagne, Angleterre, Écosse, Norvège, Danemark, Finlande). Il s'agissait, à partir d'une grille de recueil de données très précise comptant nombre d'éléments appréhendés par des observations, d'évaluer le degré de contrainte imposé aux femmes incarcérées dans chacune des institutions visitées.

L'analyse présentée dans cet article se centre, pour sa part, sur les conditions de vie des femmes en prison - prévenues et condamnées - au sein de deux institutions canadiennes considérées à titre d'exemple. L'une, la Maison Tanguay au Québec, illustre l'ancienne conception de la prison alors que l'autre, le Burnaby Correctional Center for Women en Colombiebritannique se veut le prototype de la prison moderne. Dans chacun des cas, nous procédons, premièrement, à un bref historique du développement de ces prisons et à une description relativement détaillée des conditions de vie des femmes qui y sont détenues puis nous donnons, dans un deuxième temps, un aperçu des contraintes institutionnelles particulières imposées aux prévenues, elles dont la culpabilité n'a pas encore été reconnue.

Les observations sur le terrain de ces deux institutions carcérales canadiennes pour femmes ont été menées durant l'été et l'automne 1992. Lorsque des changements importants sont survenus depuis notre visite sur les lieux, nous l'indiquons.

\section{Un portrait général des personnes incarcérées au Canada}

Au Canada, le nombre total d'admissions d'hommes et de femmes dans les institutions de détention fédérales et provinciales en 1992-1993 
(dernière année pour laquelle nous possédons des informations), se chiffrait à 148026 . Un peu moins de la moitié de ces personnes $(46,0 \%)$ étaient admises à titre de prévenus. À n'importe quel moment, au cours de la même période, le nombre de détenus (hommes et femmes) reconnus coupables et condamnés à une peine d'emprisonnement présents dans l'ensemble des institutions carcérales canadiennes s'élevait à 26477 : 14135 d'entre eux purgeaient une peine provinciale de moins de deux ans, et 12342 une peine fédérale de deux ans ou plus. Le nombre de suspects soumis à une mesure de détention provisoire, présents dans l'ensemble des institutions carcérales du Québec, à un moment donné s'établissait, pour sa part, à 5232 , soit un rapport d'un prévenu pour cinq détenus présents à un moment dans l'ensemble des institutions carcérales canadiennes. On ne trouve pas de statistiques officielles permettant d'établir avec précision le nombre de ces prévenus et détenus qui seraient des femmes. Diverses études, dont les plus importantes sont probablement celles d'Hamelin (1985) et Cousineau et al. (1986) ont néanmoins permis de constater que plus on avance dans le cours du processus pénal, plus la proportion de femmes que l'on rencontre tend à s'estomper. Ainsi, elles compteraient pour, environ, $15 \%$ des personnes appréhendées par les policiers, $10 \%$ des personnes gardées en détention provisoire, $7 \%$ des personnes condamnées à des peines provinciales (deux ans moins un jour ou moins) pour ne plus représenter que $2 \%$ des personnes se voyant imposer une peine d'emprisonnement d'une durée supérieure à deux ans.

Se servant de leur petit nombre comme justification, les deux paliers de gouvernement logent à la même enseigne les femmes reconnues coupables, et celles qui sont en attente de procès alors qu'ordinairement, dans le cas des hommes, ces deux populations sont gardées séparément. Cela est du moins vrai dans les grands centres urbains ${ }^{3}$. L'argument du petit nombre sert aussi à justifier que, sans égard au type de surveillance nécessaire pour chacune d'elles (niveau de sécurité maximum, médium ou minimum), des femmes sont indistinctement détenues sous le même toit, offrant, dans la grande majorité des cas, un niveau de sécurité maximum. Le même argument sert enfin à justifier que des condamnées, qu'elles purgent une peine de courte ou de longue durée, se trouvent toutes regroupées au sein du même centre de détention, comme à la Maison Tanguay et au Correctional Center for Women de Burnaby - ce qui, est-il nécessaire de le préciser, est par ailleurs rarement le cas pour les hommes.

3. Et moins vrai dans les régions rurales éloignées des grands centres où il n'est pas rare de voir des hommes et des femmes, prévenu(e)s et détenu(e)s se retrouver au sein de la même institution carcérale, afin de ne pas éloigner indûment les personnes incarcérées de leur milieu d'origine, et les institutions carcérales réservées aux femmes se faisant rares. Notons tout de même qu'à Montréal, le Centre de détention de Montréal (prison Bordeaux) réserve une aile aux prévenus. Cela est vraisemblablement aussi le cas d'autres prisons pour hommes, nous ne l'avons pas vérifié. 
Cette particularité de l'incarcération des femmes explique que, bien que notre étude ait initialement porté sur les femmes condamnées à une sentence d'incarcération punitive, nous pouvons nous prononcer sur les conditions d'emprisonnement auxquelles sont soumises les détenues en attente ou en cours de procès (ci-dessous nommées prévenues ou détenues provisoires), comparant ce qu'il en est pour les deux groupes : prévenues et condamnées.

\section{LA MAISON TANGUAY}

Ouverte depuis 1964, la prison pour femmes Maison Tanguay est située dans le secteur nord-ouest de l'île de Montréal. Elle a été construite en tant qu'institution provinciale réservée aux femmes présumées ou déclarées coupables, c'est-à-dire comme un établissement voué à recevoir des prévenues et des condamnées à de courtes peines de prisons (deux ans moins un jour ou moins). Toutefois, en 1972, la Maison Tanguay se voyait imposer une «double » vocation, c'est-à-dire qu'à compter de cette date, en vertu d'une entente fédérale-provinciale, elle recevra des femmes condamnées à des peines d'incarcération fédérales ou longues sentences (deux ans et plus).

\section{La population}

Principale prison pour femmes au Québec, la Maison Tanguay a admis, en 1991-1992, 2504 femmes parmi lesquelles se trouvaient 1334 prévenues $(53,3 \%)$ et 1170 femmes condamnées $(46,7 \%)$ s'étant vu imposer une sentence d'emprisonnement d'une quelconque durée. Considérant le dénombrement institutionnel quotidien, la situation se lit différemment. Sous cet angle, on constate que parmi les 151 femmes incarcérées à la Maison Tanguay, $30(19,9 \%)$ y sont gardées en attendant de connaître l'issue des procédures pénales les impliquant, alors que $121(80,1 \%)$ s'y trouvent afin de purger une sentence de quelque durée que ce soit. Cette différence de proportion entre, d'un côté, le nombre d'admissions de prévenues et de condamnées enregistrées en cours d'année et, d'un autre côté, le nombre de prévenues et de condamnées se trouvant effectivement entre les murs de la prison à un moment donné s'explique par la durée du séjour dans chacun des cas : une analyse de Statistique Canada (1993) montre, en effet, que les prévenues passent en moyenne 8,14 jours à l'intérieur des murs de la Maison Tanguay, tandis que les femmes condamnées purgent, en moyenne, 38,2 jours d'emprisonnement au sein de la même institution.

Nous notons aussi, depuis 1988 , une augmentation constante du nombre d'admissions en prison des femmes en attente de procès, augmentation compensée par une diminution de plus de la moitié de la durée de leur 
séjour. Au contraire, le nombre d'admissions de femmes condamnées à des peines d'emprisonnement tend à diminuer progressivement, alors que la durée effective de leur passage en prison augmente (Centre canadien de la statistique juridique, 1991-1992).

À la Maison Tanguay, $40 \%$ des détenues sont considérées comme des récidivistes. À noter que seules sont considérées comme des récidivistes les femmes qui n'en sont pas à leur premier séjour à Tanguay. Il s'agit donc ici plutôt d'une mesure relative aux retours multiples à l'institution que d'une véritable mesure de récidive ${ }^{4}$.

Finalement, retenons que la Maison Tanguay remplit un triple mandat, impliquant la garde de trois sous-groupes différents de détenues:

- des détenues en attente ou en cours de procès, du district judiciaire de Montréal et des environs;

- des détenues provinciales, c'est-à-dire condamnées à une peine d'emprisonnement d'une durée de moins de deux ans;

- des détenues fédérales purgeant une peine de deux ans ou plus.

Le statut (prévenue ou condamnée) motivant leur admission à la Maison Tanguay ne changera pas grand-chose, nous allons le voir, au vécu quotidien des femmes appelées à vivre une partie de leur vie au sein de cette institution.

\section{Conditions de vie à Tanguay}

Les détenues composant chacun des trois sous-groupes ci-dessus décrits attendent leur procès ou purgent leur peine, selon le cas, regroupées à l'intérieur des murs d'un même édifice principal. Des bâtiments connexes sont utilisés pour certaines des activités offertes par l'institution, pour divers ateliers et comme lieux de travail. La structure principale comprend douze «secteurs" répartis en autant d'ailes, prenant place sur deux étages. Onze des ces "secteurs" sont des unités de vie qui se répartissent en six unités de vie "normales " pour les détenues condamnées, une unité psychiatrique, une unité réservée aux prévenues, une section pour les détenues "instables», une autre pour les détenues à long terme, et encore une autre pour les détenues considérées comme «antisociales». Lors de notre observation sur le terrain, la douzième unité servait de salle de classe, où se tenaient les activités scolaires.

En 1992, l'indemnité quotidienne versée par l'État pour chacune des personnes détenues à la Maison Tanguay était de $140 \$$. On comptait environ 150 détenues dans cette institution à ce moment-là, c'est-à-dire un ratio

4. Sur cette question des retours multiples des femmes en institution carcérale, voir Imbleau (1988). 
de 1,15 détenue par cellule. On nous apprend, cependant, que l'institution souffre maintenant ${ }^{5}$ de surpopulation et que l'occupation double des cellules est devenue de plus en plus courante.

Jusqu'en 1982, nombre d'articles de toilette, d'objets personnels et de tabagie étaient gratuitement fournis aux femmes détenues. Entre 1982 et 1990, il semblerait que la gratuité ait été limitée aux produits d'utilité courante et de toilette. Depuis 1990, les détenues doivent supporter les frais de tous les effets personnels et objets de consommation courante qu'elles souhaitent sé procurer, et ce, à même leur salaire obtenu dans l'institution ou avec leur argent personnel. Les profits de la cantine, où prévenues et condamnées se procurent ces effets personnels et biens de consommation, seraient consacrés au développement de services et de programmes, nous dit-on ${ }^{6}$. Le salaire des détenues qui travaillent à l'intérieur des murs varie entre $2,50 \$$ et $3,00 \$$ l'heure; $10 \%$ des montants qui leur sont ainsi versés étaient obligatoirement placés dans leur fonds d'épargne personnel.

L'une des principales difficultés vécues par les femmes incarcérées en attendant l'issue des procédures judiciaires les impliquant ou purgeant une peine de prison vient de ce que $50 \%$ d'entre elles sont des mères qui, du fait de leur incarcération, se trouvent séparées de leurs enfants pour une période plus ou moins longue. Ainsi, alors que pour un tiers des femmes sous sentence provinciale, à la Maison Tanguay, la période de détention n'excède pas quelques mois (entre trois et six mois), lorsqu'il s'agit de femmes purgeant une sentence fédérale, cette période peut équivaloir à plusieurs années passées loin des enfants. Pour les femmes placées en détention provisoire en attendant l'issue de leur cause devant le tribunal, la période de détention qu'elles devront subir est a priori inconnue, puisqu'elles ne peuvent savoir, tenant compte des délais et des remises imposés par le tribunal, combien de temps dureront les procédures judiciaires. Elles devront donc continuellement revoir les arrangements concernant la garde des enfants, qu'elles fixeront à plus court terme possible, ne serait-ce que pour se rassurer sur leur propre situation.

Contrairement à plusieurs institutions carcérales européennes et même, depuis peu, à certaines prisons des États-Unis, dans les prisons pour femmes du Québec, on ne permet pas que les jeunes enfants vivent à l'intérieur des murs avec leur mère. Si une telle attitude peut éviter des problèmes de jalousie manifestée par les mères qui n'auraient pas l'autorisation de séjourner dans l'unité «mère-enfants» d'une prison où une telle unité existerait, il s'agit, par contre, d'une grande source d'affliction pour la majorité de celles qui sont détenues à long terme.

5. Avril 1995.

6. Résultats d'entretiens menés auprès du personnel et de la direction de l'établissement. 
Néanmoins, afin qu'elles puissent demeurer en relation avec leurs enfants, quelques femmes qui en obtiennent la permission profitent d'un lieu de visite privilégié, la roulotte, qu'elles pourront utiliser pour un jour ou deux afin d'y vivre ces quelques moments avec leur famille. Mais... d'une part, ce mode de visite n'est pas consenti à toutes les mères et, d'autre part, celles susceptibles d'y avoir droit doivent attendre leur tour.

En dehors de ce mode de visite exceptionnel, les autres visites sont limitées à quelques heures de «contact», dans le parloir traditionnel. Enfin, il arrive que certaines mères refusent toute forme de contact direct avec leurs enfants ou encore, pour différentes raisons non spécifiées, qu'elles n'y aient pas droit. Les enfants de moins de 12 ans ne sont pas fouillés au moment de leur entrée dans l'institution.

Des séances de formation aux aptitudes parentales et des cours sur l'éducation des enfants sont offerts aux mères incarcérées à la Maison Tanguay et, dans la plupart des cas, les mères sont tenues de suivre ces cours avant d'obtenir la permission de passer quarante-quatre ou soixantehuit heures consécutives avec leurs enfants dans la roulotte.

\section{Personnel de garde et autres contrôles correctionnels}

À Tanguay, $85 \%$ des 120 agents correctionnels sont des femmes. Les superviseurs d'unité doivent accomplir une double tâche de "traitement " (écoute, intervention) et de surveillance ou de contrôle. On trouve des hommes et des femmes chez ces superviseurs. Enfin, la majorité des officiers de haut rang sont des hommes.

Le système de contrôle technique (audio, vidéo, détecteurs de métal, etc.) n'est pas aussi sophistiqué qu'ailleurs (voir plus loin dans cet article ce qu'il en est en ce qui concerne le Burnaby Correctional Center for Women), mais demeure très visible, particulièrement à la réception.

Des contraintes spécifiques de temps et d'espace encadrent les femmes détenues à la Maison Tanguay. Celles-ci ont été répertoriées sur une grille d'évaluation spécifiquement conçue pour rendre compte du degré de contrainte rencontré dans les différentes institutions de notre étude ${ }^{7}$, laquelle grille de données était systématiquement remplie au moment de notre visite à chacune des 24 institutions carcérales citées dans notre méthodologie. Les données ainsi recueillies témoignent de la souplesse toute relative (ou de la présence d'une rigidité non nécessaire) des horaires et des déplacements à

7. Cinq types de contraintes ont ainsi été évaluées, soit les contraintes d'espace (y compris la limitation des déplacements à l'intérieur des murs de la prison), d'horaires (y compris la présence d'un couvre-feu), d'accessibilité aux programmes, services et activités, de liberté d'expression (y compris la possibilité de se vêtir, de se maquiller à son goût...) et de relations interpersonnelles (y compris les possibilité d'entrer en contact avec l'extérieur). 
Tanguay. Les contraintes les plus lourdes que subissent les femmes incarcérées à la Maison Tanguay ont trait à l'impossibilité de se déplacer d'un endroit à l'autre au moment choisi. Ce sont les plus importantes si on les compare, entre autres, à celles qui touchent à la liberté d'expression (associée ici à la permission de porter des bijoux ou de se vêtir selon toutes les modes !), à l'accessibilité aux services de santé et aux modes de relations interpersonnelles. Elles sont néanmoins moins prégnantes que celles observées dans d'autres prisons pour femmes, notamment, comme nous le verrons, au Correctional Center for Women de Burnaby.

\section{Activités et programmes}

Ce qui faisait - et fait encore - défaut de manière évidente à la Maison Tanguay, ce sont des programmes de travail, d'éducation et de loisirs, intéressants, modernes, à jour. Par exemple, dans cette institution, les femmes confinées à l'édifice principal peuvent s'adonner uniquement à des tâches de ménage et d'entretien. Celles qui ont accès aux bâtiments adjacents peuvent participer à un atelier de petits travaux à la chaîne ou à l'exécution de contrats de couture. Un bon nombre de femmes peuvent encore se consacrer aux tâches d'usage dans une buanderie, elle aussi située dans un bâtiment isolé.

Quant aux programmes d'études, les femmes se voient offrir les possibilités limitées de suivre des cours de niveau secondaire et de recevoir une formation en bureautique (à moins qu'elles ne s'inscrivent à des cours par correspondance).

Celles qui auraient besoin de services thérapeutiques peuvent s'inscrire à un groupe des Alcooliques anonymes. En outre, une psychologue est disponible durant les jours de semaine aux fins de consultations. Il semble, cependant, qu'en raison d'une longue liste d'attente, plusieurs détenues endurent leurs problèmes et passent outre le traitement.

L'absence d'une variété raisonnable de programmes de travail et de formation se fait sentir plus cruellement à la Maison Tanguay que partout ailleurs parmi les institutions que nous avons visitées; on ne peut en effet que constater combien les occupations qui y sont offertes sont ennuyeuses, vaines et peu reliées à une vie éventuelle hors les murs. Devant cette carence importante, nous nous sommes questionnée sur l'équité vis-à-vis des détenues, notamment par comparaison à : $a$ ) ce à quoi les femmes ont accès hors prison; $b$ ) ce qui existe dans les institutions carcérales pour hommes.

\section{Les détenues en attente ou durant procès, ou détenues provisoires}

En tant que détenue soumise à une mesure de détention provisoire pour une partie ou la durée des procédures judiciaires l'impliquant à titre d'inculpée, 
une femme n'a pas accès au travail rémunéré, qui, bien que limitê, est néanmoins offert à la Maison Tanguay aux condamnées. Les prévenues qui se voient refuser le droit de franchir les limites de l'édifice principal de la prison n'ont donc pas non plus accès aux activités tenues dans les édifices adjacents. Elles ne peuvent donc par conséquent pas travailler ni à la buanderie ni à l'atelier de couture, qui sont situés dans des bâtiments isolés. Il est sous-entendu que le fait de se rendre dans les lieux situés en dehors de la structure principale de la prison n'est pas sécuritaire parce que ces sites sont dépourvus de la surveillance requise pour et par les femmes prévenues, nous reviendrons sur ce point. Celles-ci se voient donc limitées au seul choix des tâches d'entretien de base: nettoyage, corvées de cuisine ou autres travaux monotones. Les prévenues reçoivent néanmoins en compensation de ces tâches ménagères des salaires comparables à ceux offerts aux femmes d'ores et déjà condamnées.

À la Maison Tanguay, les femmes détenues durant la tenue de leur procès peuvent suivre des cours et ont la permission d'intégrer la population générale de la prison, encore une fois tant que cette intégration a lieu à l'intérieur des limites de l'édifice principal. En d'autres mots, elles peuvent manger à la cafétéria, faire de l'exercice au gymnase et rencontrer d'autres détenues.

Être détenue à la Maison Tanguay dans l'attente de son procès comporte encore d'autres désavantages que ceux que nous venons d'énumérer. En égard à la population des prévenues, la philosophie de la prison repose sur la nécessité d'une surveillance supplémentaire par rapport aux condamnées. Certaines femmes en attente de procès se retrouvent en détention pour la première fois et, de ce fait, ne connaissent pas les codes et les moyens de survie à l'intérieur des murs. Elles doivent donc être protégées des autres. Dans d'autres cas, il s'avère que la détention provisoire a été imposée pour des raisons de sécurité publique, parce que la prévenue est soupçonnée d'être l'auteure de crimes graves. On considère alors que les autres détenues doivent être protégées. Enfin, certaines vivront les périodes de manque associé à des habitudes récentes de consommation abusive de drogues subitement interrompues. Il est également jugé plus sécuritaire d'isoler cette catégorie de détenues. Enfin, dans tous les cas, ces femmes incarcérêes sans connaître l'issue des procédures judiciaires auxquelles elles sont soumises souffrent d'un état de stress intense du fait même de cette incertitude face aux conclusions judiciaires sur la cause et face à l'éventualité de la peine. Selon le personnel de la prison, toutes ces conditions particulières font des détenues provisoires une clientèle extrêmement exigeante, notamment sur le plan de la sécurité.

Signalons enfin que les conditions de détention provisoire sont particulièrement difficiles pour les femmes qui ont des enfants. La période de 
séparation entre la mère et ses enfants est impossible à déterminer avec le moindrement de précision, étant donné qu'elle est fonction du déroulement des procédures judiciaires. Alors, non seulement la mère devra continuellement revoir les arrangements pris à court terme pour la garde des enfants, phénomène précédemment signalé, mais elle devra aussi envisager le pire, c'est-à-dire la possibilité de voir cette période de détention provisoire se transformer en période plus ou moins longue d'incarcération punitive nécessitant une toute autre forme d'arrangement. En outre, durant la période de sa détention provisoire, comme il ne lui est pas permis d'avoir accès à l'extérieur de l'édifice principal de la prison, la prévenue se verra refuser le droit d'utiliser la roulotte aux fins de visites familiales.

Ainsi, considérant les limites particulièrement contraignantes, notamment quant à l'accès au travail et aux activités de loisirs, imposées aux prévenues en attente de procès à la Maison Tanguay — pour une période pouvant s'étendre de un jour à plus d'un an - on peut facilement imaginer que cette période d'attente, angoissante pour plusieurs, s'avérera non seulement un long moment d'ennui pour beaucoup, mais que pour certaines, il s'agira d'un moment tragique de leur vie.

\section{LE BURNABY CORRECTIONAL CENTER FOR WOMEN}

Le Burnaby Correctional Center for Women est situé dans la banlieue de Vancouver, en Colombie-Britannique. Cette institution, érigée en 1991 , a remplacé les prisons pour femmes de "Lakeside " et de «Maple Creek". Sa structure moderne et hautement technologique, au sein d'un vaste décor rural, offre suffisamment d'espace pour accueillir plus de 170 femmes. Au moment de notre visite, la population carcérale présente au sein de l'institution était d'environ 145 détenues. On est loin ici d'une situation de surpopulation. De fait, parce que certaines ailes de la prison demeuraient vides (ce qui était le cas d'au moins trois ailes pouvant chacune accueillir une dizaine de détenues) en 1994, les services correctionnels de la ColombieBritannique ont finalement pris la décision de permettre à l'institution $d^{\prime}$ ouvrir ces ailes à un certain nombre de jeunes hommes ${ }^{8}$ peu criminalisés devant purger une courte peine. Au moment de notre visite, cette pratique d'accueillir des hommes au Burnaby Correctional Center for Women n'avait pas encore cours, de sorte qu'il nous est impossible d'analyser les changements qu'une telle situation risque de générer au sein de cette prison originellement construite pour y recevoir des femmes et réservée à cet usage. En 1995, la prison détient 17 hommes en moyenne, pour n'importe quel jour donné. Il sera indéniablement intéressant de suivre ces développements

8. Les notions de «jeune homme" et de "peu criminalisés» demeurent fort vagues. 
qui représentent une première, à tout le moins dans l'histoire des prisons au Canada ${ }^{9}$.

\section{Les conditions de vie}

Parce que le Burnaby Correctional Center for Women loge dans un édifice nouvellement construit, il se démarque des autres institutions que nous avons visitées par sa propreté, son modernisme, son atmosphère de tranquillité et son architecture relativement innovatrice.

Diverses caractéristiques de cette architecture ne sont cependant pas sans comporter certains inconvénients. Par exemple, cette institution n'offre pas de cafétéria commune. À l'heure des repas, le personnel se déplace à travers la prison avec chariots et plateaux contenant les repas déjà préparés et les distribue à chaque unité, comme dans un hôpital. Certaines femmes se plaignent que leurs repas arrivent froids. En lieu et place de la cafétéria, qui dans plusieurs institutions sert de lieu de rencontre, on trouve au Burnaby Correctional Center une rotonde. Il s'agit d'une vaste aire ouverte surplombée de puits de lumière et agrémentée de plantes; on y trouve des bancs où l'on peut s'asseoir et discuter avec des détenues d'autres ailes (autrement, comme dans les autres prisons, les détenues sont cantonnées dans l'aile qui leur est assignée). La rotonde est entourée par les salles de classe, la chapelle, et la bibliothèque. C'est aussi à partir de la rotonde que l'on accède aux différents lieux de travail et d'activités de loisirs, ainsi qu'aux aires extérieures. Cet arrangement rend impossible toute forme d'intimité et permet de suivre les allées et venues vers et hors de chacun de ces lieux. Chaque déplacement, chaque geste, est ainsi «public».

\section{Les activités, les programmes de travail et les services}

On trouve au Burnaby Correctional Center for Women, un grand atelier de couture où les femmes qui le désirent peuvent travailler à la confection des traditionnels uniformes verts portés par les hommes en prison. Au moment de notre visite, l'atmosphère y était tendue, et peu de femmes le fréquentaient.

Le centre propose aussi un atelier de coiffure où, encore une fois, les femmes détenues qui le souhaitent apprennent les techniques de la coupe, de la teinture et de la permanente. Ce lieu ressemble à n'importe quel salon de coiffure d'une petite communauté. Il offre donc, dans une certaine

9. Il arrive par contre, relativement couramment, surtout dans les petites prisons situées dans des régions éloignées des grands centres, qu'on retrouve quelques femmes, incarcérées en attendant l'issue de leur procès ou pour de courtes peines d'emprisonnement, au sein d'institutions originellement conçues pour recevoir des hommes et normalement réservées à cet usage. Il s'agit alors d'éviter que ces femmes soient trop éloignées de leur milieu de vie habituel, le temps que dure leur incarcération. 
mesure, l'illusion d'œuvrer comme n'importe qui à l'extérieur des murs. Toutefois, à l'issue de leur formation ces femmes n'obtiendront pas de diplôme.

Diagonalement opposée à l'atelier de couture se trouve une grande boutique de fleurs où des femmes apprennent les soins des plantes et des fleurs naturelles, ainsi que les techniques d'arrangement créatif de fleurs séchées et de soie. Lors de notre visite, cet atelier nous a paru vivant. On nous a confié que l'animatrice de l'atelier fait en sorte d'obtenir des contrats de travail de l'extérieur et qu'une part des profits ainsi générés est versée au fonds des détenues. Celles-ci voient ainsi le fruit de leur travail leur rapporter quelques dividendes.

Un autre programme de travail, rarement vu ailleurs dans les institutions carcérales canadiennes, mais disponible au Burnaby Correctional Center for Wornen est l'Entraînement K-9; il s'agit de dresser des chiens à diverses fins (propreté, défense...). Cette activité se tient à l'extérieur des murs, dans une aire néanmoins fermée par tout un dispositif de clôtures. Les membres du personnel chargés de cet atelier apprennent aux femmes les méthodes de dressage des chiens. Ces derniers proviennent de la Société protectrice des animaux et, une fois entraînés, sont vendus à des personnes habitant dans la région de Vancouver. Encore une fois, les femmes voient leur travail porter fruit.

Jouxtant l'aire réservée au K-9 se trouvent les installations nécessaires au programme de formation en horticulture où les femmes peuvent apprendre des techniques et procédures spécialisées de culture de légumes et de plantes. Lors de notre visite, plusieurs femmes s'affairaient aux soins de l'une ou l'autre variété de plantes que l'on retrouve dans la serre et l'atelier attenant. Les plantes, comme les fleurs de la boutique de fleuriste, sont vendues lors d'événements particuliers et de fêtes spéciales, tout au long de l'année. Une détenue nous confie qu'elle se sent rajeunie dans ce site de culture où l'on peut, d'une certaine façon, sentir la vie.

Un dernier atelier est, pour sa part, situé en dehors de la prison proprement dite, non loin des sites d'horticulture et du K-9. Il s'agit d'un atelier de poterie qui sert de lieu d'expression de la créativité, soit dans un cadre d'apprentissage, soit dans un contexte de loisirs. Comme dans le cas des produits d'horticulture et des fleurs de la boutique, ces objets d'artisanat peuvent être vendus lors d'événements spéciaux et de fêtes.

Le travail au Burnaby Correctional Center for Women est rémunéré en fonction d'une échelle de salaires comprise entre 3,00\$ et 7,00\$ par jour, selon la nature du travail. On a pu constater que certaines détenues, quand approche le terme de leur détention, font du travail supplémentaire afin de gagner plus d'argent. 
Enfin, bien que la prison soit dotée d'un gymnase moderne, propre et entièrement équipé, pour des raisons inexpliquées, celui-ci sert peu. En effet, rares sont les détenues qui s'y adonnent au basket-ball, au volley-ball, au tennis, au badminton ou autres sports du genre. Les sports individuels, et pour certains non traditionnels pour jes femmes, comme la levée de poids, trouvent davantage preneur. Besoin de se démarquer? C'est fort possible.

\section{La surveillance et le contrôle}

Le centre de contrôle est certainement l'installation la plus impressionnante de l'institution. Les employées qui travaillent dans cette «station» ont le pouvoir d'ouvrir et de fermer électroniquement plus de 100 portes, intérieures et extérieures, et maîtrisent au moins soixante-quinze caméras surveillant les allées, venues, et activités des détenues. Un agent correctionnel peut ainsi contrôler électroniquement plus de 1000 ouvertures de portes par jour. En outre, depuis cette station de contrôle, toute personne - détenue ou membre du personnel, dans l'institution ou en dehors - peut être visualisée sur écran. Tous les espaces de l'institution (les corridors, la salle des visites, la rotonde, l'entrée de la prison, la cuisine, le gymnase et ainsi de suite, les cellules individuelles des détenues exceptées), de même que ses limites périmétriques sont saisies sur ces véritables «scanographies ". Les visiteurs, les détenues et le personnel savent que lorsqu'ils vont et viennent dans l'institution, chacun de leurs déplacements, chacun de leurs gestes sont enregistrés. On comprend que ce type de "contrôle " représente un facteur de stress, autant pour le personnel que pour les détenues, comme on nous l'a confié ${ }^{10}$.

\section{Les détenues en attente de procès}

Les détenues en attente de procès composaient au moins $20 \%$ de la population des femmes emprisonnées au Burnaby Correctional Center for Women au moment de notre visite, une bonne proportion de ce $20 \%$ étant constituée de femmes qui n'en étaient pas à leur premier séjour au sein de cette prison. Selon la personne en charge de l'institution (mars 1995), les détenues avant procès sont mêlées à la population générale de la prison, même s'il existe une unité réservée aux prévenues. Cependant, si une femme est jugée "naïve» ou n'est pas considérée comme "street smart», ou encore si l'administration de la prison croit qu'il y a quelque raison de la protéger des autres détenues, elle sera gardée à l'écart de la population générale. La directrice de l'institution indique qu'elle vise présentement ${ }^{11}$ à faire disparaître l'existence d'une politique de «séparation» entre les

10. Résultats d'entretiens avec des détenues et des membres du personnel lors de notre visite.

11. Avril 1995. 
prévenues et les condamnées et essaie de «mixer " le plus possible ces deux groupes.

Ici, comme à la Maison Tanguay, les femmes prévenues peuvent attendre jusqu'à un an la tenue de leur procès. Mais cette procédure arrive à terme, en moyenne, avant six mois révolus. La règle générale veut que plus un cas est compliqué, plus longue sera l'attente avant le procès. De telles circonstances augmentent la possibilité que la prévenue plaide coupable, et ce, seulement afin de mettre un terme à cette période d'incertitude et de stress vécue difficilement ${ }^{12}$.

Ces détenues peuvent cependant profiter d'un programme de vie passablement varié (rien à voir ici avec la Maison Tanguay). Elles ont le droit d'accéder à un emploi, le jour même suivant leur arrivée; les salaires qui leur sont offerts se comparent à ceux versés aux condamnées. En outre, au Burnaby Correctional Center for Women, les prévenues ont accès aux activités, à la scolarisation et aux traitements médicaux. De fait, la directrice du centre nous indique ${ }^{13}$ que, fondamentalement, il n'y aurait pas de différences de traitement au sein de cette institution entre les prévenues et les condamnées. Dans certains cas, les prévenues seront même avantagées. Ainsi, comme il n'y a pas d'obligation de travailler pour les détenues en attente de procès, celles-ci ne se retrouvent pas enfermées à clé si elles refusent de travailler. Par contre, les condamnées, elles, sont écrouées si elles omettent de se rendre au travail sans excuse valable.

Nonobstant le fait qu'on trouve au Burnaby Correctional Center for Women un programme d'activités beaucoup plus varié qu'à la Maison Tanguay, cette institution présente un degré de contrainte des plus élevés en regard de l'espace, du temps, des relations interpersonnelles et de la liberté d'expression. En d'autres mots, dans cette institution, une détenue a moins de liberté pour «aller et venir » comme il lui plaît, et doit se plier à un horaire quotidien très strict. Les contraintes qui affectent les relations interpersonnelles y sont aussi multiples: la distance que les familles des détenues doivent parcourir et la durée limitée des visites, la difficulté pour les détenues de rencontrer d'autres détenues logeant dans des unités différentes de la prison, autrement que sous la rotonde où s'exerce une surveillance de tous les instants, limitent grandement les possibilités de contacts interpersonnels le moindrement intimes. Nous avons aussi noté que la liberté d'expression des femmes était limitée étant donné les exigences de l'institution en ce qui a trait aux vêtements, aux bijoux, aux chaussures, au maquillage, etc. Il n'en demeure pas moins que cette institution offre aux femmes soumises à une mesure de détention provisoire de plus grandes possibilités en ce qui a trait aux programmes, services et activités leur étant accessibles.

12. Ȧ ce sujet voir, entre autres, Gravel et Cousineau, 1989.

13. Conversation téléphonique avec la directrice de la prison en mars 1995. 
Mais cela est-il suffisant pour contrebalancer l'atmosphère de contrôle incessant dans laquelle se réalisent ces activités? La question reste ouverte.

\section{Une explication patriarcale des contraintes pénales imposées aux femmes}

Cherchant une théorie qui nous aiderait à comprendre les contraintes auxquelles sont soumises les femmes en prison (prévenues et détenues confondues), en ce milieu des années 1990 , nous avons retenu la thèse de MacKinnon (1989) qui s'appuie sur l'idée que le droit et les institutions d'État sont à caractère mâle. En fait, cette prémisse de l'auteure, à savoir une société patriarcale et «des lois et institutions mâles ${ }^{14}$ ", nous permet de poursuivre notre travail de compréhension du raisonnement qui se lit derrière plusieurs des contraintes pénales spécifiquement rencontrées dans les prisons pour femmes et imposées tant aux prévenues qu'aux condamnées, comme celles mentionnées plus haut : contraintes sur le temps, l'espace, sur l'accessibilité aux programmes, aux activités et aux services. Dans certains cas, les structures imparties aux femmes sont absolument identiques à celles créées par les hommes et réservées aux hommes, alors que d'autres fois, leur spécificité même les rend pires. C'est notamment le cas, à notre avis, des deux prisons pour femmes que nous étudions ici, la Maison Tanguay et le Burnaby Correctional Center for Women, qui gardent des populations exigeant différents niveaux sécuritaires (maximum, médium et minimum) - et ceci aussi bien pour des prévenues que pour des condamnées - dans des conditions de surveillance maximales.

Matériellement, les femmes détenues sont souvent logées dans des prisons jugées trop petites ou vêtustes pour les hommes et qui manquent d'équipement moderne et de locaux appropriés pour une variété de programmes intéressants et enrichissants. C'est ce qui ressort de notre analyse de la Maison Tanguay où, par exemple, l'équipement - comme les machines à coudre, les imprimantes et les ordinateurs - est largement dépassé. En outre, dans le choix des programmes de travail et d'activités offerts aux femmes incarcérées, particulièrement à la Maison Tanguay, il est clair qu'on ne s'est pas préoccupé des besoins des femmes ni de leur «culture » particulière.

Le fait même que l'institution, d'un côté, isole les individus de "l'extérieur», ne permettant qu'un minimum de contacts avec la famille, les amis et l'environnement social, alors que, de l'autre côté, elle entasse les prévenues et les détenues à «l'intérieur», crée une situation où les problèmes quotidiens, les moindres désagréments et événements un tant soit peu problématiques sont amplifiés. De plus, les femmes en prison 
doivent faire face à de nouvelles situations comme les repas collectifs, les visites collectives, la difficulté à se procurer de l'argent, le fait de devoir payer tous leurs appels téléphoniques, qu'ils soient locaux ou interurbains, et le reste. Enfin, l'intimité est virtuellement impossible pour les femmes en prison, et notons qu'il n'est pas rare qu'elles demandent à être isolées (en ségrégation) afin de trouver un peu de solitude.

La privation sensorielle, une surveillance constante (particulièrement à la prison de Burnaby), la cohabitation obligée et la vie de prison en ellemême créent, dans plusieurs cas, des états de morbidité, et rendent nécessaires les programmes thérapeutiques. Il existe différentes thérapies, comme la gestion de la colère, l'apprentissage d'aptitudes sociales et autres de même type, auxquelles les femmes ont accès, mais nous n'avons aucunement évalué l'adéquation de ces «traitements » pour elles. Plus encore, peu de thérapies pourraient se vanter de rendre aux femmes leur autonomie («empowering") et de les aider véritablement à combattre l'oppression, bien que quelques conseillères du Burnaby Correctional Center nous aient affirmé utiliser une approche féministe dans le traitement des femmes emprisonnées.

Dans d'autres cas, comme dans les programmes et services, la diversité des équipements s'avère nettement insuffisante. Dans d'autres cas encore, comme en ce qui concerne les contraintes sur le temps et la fréquentation des espaces, les règles, nous semble-t-il, sont inutilement sévères.

Quant aux programmes et aux activités offerts aux femmes en prison, la recension des écrits nous a permis de voir que "le constat du petit nombre " est la seule rationalisation utilisée pour expliquer l'absence relative de programmes intéressants dans les prisons pour femmes. Peut-on la considérer comme valable?

Afin de saisir plus en profondeur le sens des contraintes spécifiques auxquelles sont assujetties les femmes en prison, nous nous sommes tournée vers des féministes comme Bertrand (1969, 1984, 1992), Biron (1992), Carlen (1990), Daly (1989), Heidenshohn (1985, 1992), entre autres. Ces auteures féministes s'entendent pour dire que les femmes doivent continuer de se battre pour l'obtention de droits égaux et que la fin de la discrimination à l'égard des femmes passe particulièrement par des changements d'ordre légal. De plus, on doit étudier le contexte social patriarcal et analyser son impact de façon critique. En particulier, Bertrand (1992) affirme que c'est d'abord la criminalisation des femmes qui doit être étudiée, dans une perspective historique, par les auteures féministes et que cette étude doit être poursuivie, notamment du point de vue de la construction des problèmes sociaux qui impliquent les femmes. Précisément, nous devons étudier l'histoire des processus de criminalisation qui ont affecté particulièrement les femmes, telles la criminalisation de la maternité hors mariage, de 
la prostitution ou de l'infanticide, afin de comprendre les contrôles contemporains sociaux et pénaux exercés envers les femmes.

\section{CONCLUSION}

Jusqu'ici, nous n'avons pas trouvé d'argumentation solide et valide qui justifierait la nécessité, voire la possibilité, d'imposer intégralement la prison aux femmes. Nous pouvons affirmer que les contraintes de temps et d'espace infantilisent les détenues et que, comme les prisons pour femmes offrent des programmes de travail et d'éducation limités, elles ont pour effet de réduire sérieusement les conditions d'interrelation entre les femmes prévenues et détenues et celles de l'extérieur. Doit-on en conclure que la finalité de la prison est de déshabiliter les détenues?

Il y a peu de femmes incarcérées au Canada, tant sous juridiction provinciale que fédérale mais, parmi elles, le nombre de femmes prévenues est loin d'être insignifiant. Celles-ci, rappelons-le, n'ont pas été déclarées coupables. Or, les conditions d'emprisonnement de ces femmes sont aussi mauvaises, sinon pires, que celles des condamnées à une peine d'emprisonnement.

Nous ne pouvons ignorer le surplus de surveillance imposé aux détenues en attente de procès dans certaines institutions pour femmes au Canada. L'absence de classification de "sécurité " fait en sorte que peu importe qu'elles nécessitent une surveillance de type minimum, médium ou maximum, les prévenues comme les condamnées se voient toutes soumises aux mêmes restrictions quant à leur liberté de mouvement, aux espaces qui leur sont accessibles, aux privilèges, et à l'accès aux programmes, activités et services. En effet, les différences entre une femme condamnée à la prison et une femme détenue dans une unité de prévenues (lorsque de telles unités existent) sont, en toute honnêteté, pratiquement nulles. Quand des différences sont manifestes, elles le sont, très généralement, au détriment des femmes détenues avant procès.

À la lumière de ce qui vient d'être exposé, nous devons maintenant nous questionner sur des façons moins pénalisantes de gérer l'actuelle surpopulation des prisons pour femmes (rencontrée notamment à la Maison Tanguay). Cette situation a été et continue d'être une question urgente pour les personnes condamnées à purger leur peine au sein d'une institution pénale, mais c'est un problème encore plus sérieux dans le cas des détenues qui n'ont pas encore été jugées et qui, pendant ce temps, subissent des contraintes pénales très lourdes, étant souvent considérées comme des détenues de second ordre, étant donné le caractère présumément temporaire de leur situation. Rappelons toutefois que certaines prévenues attendront un an la tenue de leur procès à l'ombre des murs de la prison. Il y a là, certainement, tout un questionnement à poursuivre. 


\section{RÉFÉRENCES}

BERTRAND, Marie-Andrée, (1984), «Femmes et Justice : Problèmes de l'intervention ", Criminologie, Les presses de l'Université de Montréal. Montréal, Québec, vol. $16, \mathfrak{n}^{\circ} 2$, pp. $77-88$.

BERTRAND, Marie-Andrée, (1969), "Self-image and delinquency: A contribution to the study of female criminality and women's image ", Acta criminologica : Études sur la conduite anti-sociale, vol. 2., pp. 71-144.

BERTRAND, Marie-Andrée, (1994), «Le pouvoir des théories féministes dans la reconsidération radicale des théories du contrôle social ", dans Canada : Theoretical Discourse / Discours théoriques, édité par Terry Goldie, Carmen Lambert et Rowland Lorimier, Association for Canadian studies, Montréal, pp. 49-74.

BIRON, Louise, (1992), "Les femmes et l'incarcération, le temps n'arrange rien", Criminologie, Les presses de l'Université de Montréal, Montréal. Québec, XXV, $n^{\circ} 1$, pp. $119 \cdot 134$.

CARLEN, Pat, (1990), Alternatives to women's imprisonment, Open University Press, Milton Keynes.

CENTRE CANADIEN DE LA STATISTIQUE JURIDIQUE (1991-1992), Services correctionnels pour adultes au Canada. Canada : Statistique Canada.

CENTRE CANADIEN DE LA STATISTIQUE JURIDIQUE (1993), Les services correctionnels au Canada : fait saillants de 1991-1992. Canada: Statistique Canada.

COUSINEAU, Marie-Marthe, LABERGE, Danielle, THÉORÊT, Bruno, Prisons et prisonniers: Une analyse de la détention provinciale durant la dernière décennie. Les cahiers du GRAPPP, cahier n 1, Montréal: Université du Québec à Montréal et Centre international de criminologie comparée.

DALY, Kathy, (1989), «Rethinking judicial paternalism : gender, work-family relations and sentencing », Gender and Society, vol. 3, n'1, pp. 9-36.

GRAVEL, Sylvie, COUSINEAU, Marie-Marthe (1989), La pratique de la négociation de plaidoyer au palais de justice de Montréal, Montréal : Centre international de criminologie de Montréal.

HAMELIN, Monique (1985), Les québécois et les québécoises pénalisés, Montréal : Université de Montréal.

HEIDENSOHN, Frances, (1992), "Gender and Crime », sous presse.

HEIDENSOHN, Frances, (1985), Women and crime, MacMillan Publishers Ltd, Londres.

IMBLEAU. Monique, (1990), Profession détenue: analyse de la population des femmes réincarcérées dans les prisons du Québec, Les cahiers du GRAPPP, cahier $n^{\circ}$ 6, Montréal : Université du Québec à Montréal et Centre international de criminologie comparée.

MACKINNON, Catherine, (1989), Toward a feminist theory of the State, Harvard University Press, Cambridge, Massachusetts.

SOLLICITEUR GÉNÉRAL, CANADA, (1992), Faits et chiffres sur les services correctionnels au Canada, ministêre des Approvisionnements et Services, Canada. 